Check for updates

Cite this: RSC Adv., 2018, 8, 5714

Received 8th November 2017

Accepted 16th January 2018

DOI: 10.1039/c7ra12222e

rsc.li/rsc-advances

\section{A label-free and sensitive photoluminescence sensing platform based on long persistent luminescence nanoparticles for the determination of antibiotics and 2,4,6-trinitrophenol $\dagger$}

\author{
Xi Zhang, ${ }^{a} \mathrm{Na}-$ Yan Xu, ${ }^{a}$ Qiong Ruan, ${ }^{a}$ Dan-Qing Lu, ${ }^{b}$ Yun-Hui Yang ${ }^{\star a}$ \\ and Rong $\mathrm{Hu}(\mathrm{D}$ *a
}

\begin{abstract}
The rapid detection of pollutants with high sensitivity and selectivity is of considerable significance for security screening, environmental safety, and human health. In this study, we prepared persistent luminescence nanoparticles (PLNPs) and constructed a label-free sensor for sensitive and selective detection of pollutants in real samples and test papers. Following excitation, PLNPs could store absorbed light energy and release it in the form of luminescence. Compared with a fluorescence-based technique, a PLNPs-based measurement could effectively avoid background interference. Under optimal conditions, the limit of detection for TNP was found to be $10 \mathrm{nM}$, while for an antibiotic it was $5 \mathrm{nM}$. The nanoprobe was successfully applied for the detection of pollutants in real samples including milk and Dianchi Lake water samples. Due to the long-lasting afterglow nature of PLNPs, the signal-to-noise ratio could be greatly increased in complex real samples. By hand-writing with TNP solution as ink on filter paper, the photoluminescence (PL) of the part stained with TNP was immediately quenched. Moreover, after direct exposure under a UV lamp for $10 \mathrm{~min}$ and without further excitation, the luminescence of the test paper was investigated to avoid interferents. This PLNP material could be potentially employed as a multiresponsive luminescent sensor. In addition, these easy-to-use visual techniques could provide a powerful tool for a convenient POC assay of organic pollutants.
\end{abstract}

\section{Introduction}

The rapid and selective detection of pollutants is of considerable significance for security screening, environmental safety, and human health. Antibiotics are extensively employed for the treatment of bacterial infections in animals and humans. ${ }^{1,2}$ It is reported that the total antibiotic usage in 2013 was approximately 162000 tons in China. ${ }^{3,4}$ Moreover, the abuse of antibiotics could cause the accumulation of high levels of antibiotic residues, so they are regarded as a class of important organic pollutants. ${ }^{1,5,6}$ Similarly, nitroaromatics such as 2,4,6-trinitrotoluene (TNT) and 2,4,6-trinitrophenol (TNP or picric acid) are widely used as explosives, which are also considered as undesirable organic pollutants in waste water. ${ }^{7,8}$ These chemicals are

${ }^{a}$ College of Chemistry and Chemical Engineering, Yunnan Normal University, Kunming 650092, Yunnan, P. R. China.E-mail: hudierong_168@163.com; yyhui2002@aliyun. com; Tel: +86-871-65941087

${ }^{b}$ Molecular Science and Biomedicine Laboratory, State Key Laboratory of Chemo/ Biosensing and Chemometrics, College of Chemistry and Chemical Engineering, Collaborative Innovation Center for Molecular Engineering for Theronastics, Hunan University, Changsha, 410082, China

$\dagger$ Electronic supplementary information (ESI) available. See DOI: $10.1039 / \mathrm{c} 7 \mathrm{ra} 12222 \mathrm{e}$ highly poisonous and difficult to degrade by nature. Apart from being highly explosive, TNP is frequently used in dyes, fireworks, matches and leather industries. It can cause severe irritation, skin allergy, dizziness, nausea, as well as liver and kidney damage. To address these issues, various luminescent sensors with high sensitivity as well as simplicity have been developed for the sensing of these specific pollutants. ${ }^{9-11}$ However, these reported luminescent methods often suffer from some limitations such as low detection limits or poor signal-to-noise ratio from autofluorescence. Therefore, there is an urgent need to explore new materials or reagents for the detection of antibiotics and nitroaromatic pollutants.

Recently, substantial efforts have been made worldwide to resolve these problems. To date, numerous nanomaterials have been synthesized for the sensitive and selective detection of pollutants. Li et al. ${ }^{1}$ and Han et al. ${ }^{6}$ designed a class of porous metal-organic frameworks (MOFs) for the selective detection and removal of antibiotics and nitroaromatics. Xing et al. developed amino-functionalized MOFs for the detection of TNP. ${ }^{12}$ Unfortunately, MOFs are not stable in water during longterm storage in the water. Most of the reported MOFs perform detection of TNP is in the organic/organic-water mixture phase ${ }^{13-15}$ which might not be applicable for real sample 
detection, such as biological samples. Recently, Xian et al. ${ }^{\mathbf{1 6}}$ synthesized a polyimide covalent organic framework for the detection of TNP. Wu et al. ${ }^{17}$ and Huang et al. ${ }^{18}$ synthesized carbon nanodots for TNP detection. Chen et al. ${ }^{19}$ reported a TNP detection strategy based on fluorescent silicon nanoparticles. Although good performances have been achieved, these methods usually suffered from low sensitivity. Thus, developing a new sensing platform for pollutants in water with high sensitivity is still necessary.

Lanthanides $\left(\mathrm{Ln}^{3+}\right)$-doped upconversion nanoparticles have been rapidly developed in the past decade. These particles are suitable for use as alternatives to conventional downshifting luminescent bioprobes, such as quantum dots or organic fluorescent dyes, because of their long photoluminescence (PL) lifetimes and low toxicity. ${ }^{20-22}$ Inspired by the unique properties of upconversion nanoparticles, in this paper, a new method for the synthesis of persistent luminescence nanoparticles (PLNPs) is presented. PLNPs are one of the important light-light transformation and energy-saving materials, ${ }^{23-27}$ which could store the absorbed light energy and release it in the form of luminescence following excitation. The photoluminescence lifetime of PLNPs is of the order of several hours or longer, ${ }^{24-28}$ while common organic dyes and biocompounds are of the order of nanoseconds. ${ }^{29,30}$ These PLNPs phosphors might open numerous technologically important applications in security, solar energy utilization and medical diagnosis.

Compared with fluorescence-based techniques, PLNPsbased measurements could effectively avoid background interference. They exhibit less autofluorescence, have reduced light scattering and are less affected by the environment, such as fluctuations in oxygen concentration and the emission spectra of other heavy metal complexes. These properties make PLNPs promising alternatives for bioimaging and biosensor applications. For example, Yan et al. ${ }^{24}$ reported a fluorescence resonance energy transfer (FRET) inhibition assay for $\alpha$-fetoprotein (AFP) detection using functionalized PLNPs. Their long-lasting afterglow nature allows detection and imaging without external illumination, thus eliminating autofluorescence and light scattering from biological matrices encountered under in situ excitation. Thus, PLNPs would appear to be a favorable platform for the construction of detection methods for pollutants. However, these materials have rarely been explored in biosensor applications such as the detection of antibiotics and explosives in the environment. Therefore, it is of great interest to use these PLNPs for the detection of antibiotics and organic explosives due to their low autofluorescence and reduced light scattering, which could effectively avoid background interference.

Herein, we constructed a label-free sensor for sensitive and selective detection of organic pollutants (Fig. 1). There was a wide overlap between the absorption spectra of the pollutants and the photoluminescence (PL) excitation spectra of the PLNPs. Thus, the luminescence of PLNPs could be quenched by antibiotic and nitroaromatic pollutants. Consequently, quantitative and selective analysis of the antibiotic and TNP could be achieved under optimal conditions. The nanoprobe was

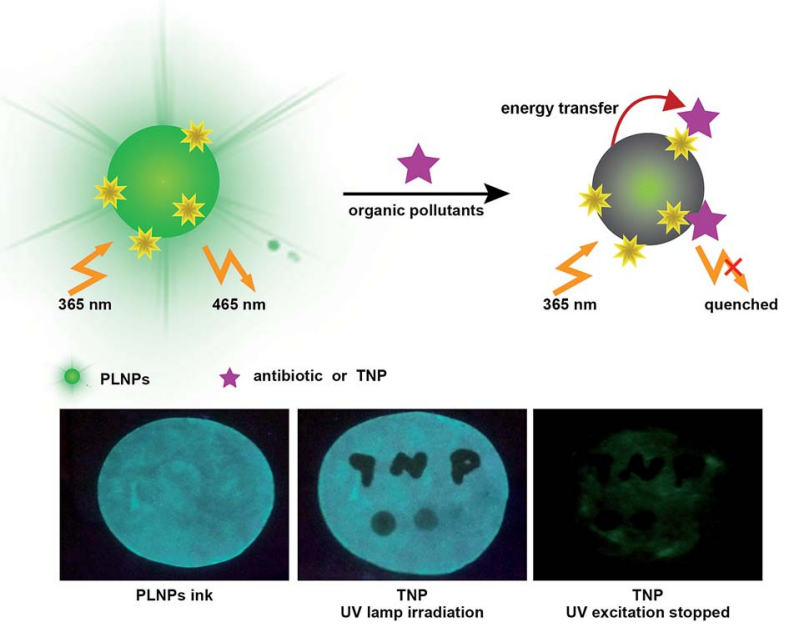

Fig. 1 Schematic illustration for the sensitive and selective detection of organic pollutants based on the PLNPs nanoprobe.

successfully applied for the detection of pollutants in real samples including milk and Dianchi Lake water samples. Due to the long-lasting afterglow nature of PLNPs, the signalto-noise ratio could be greatly increased in complex real samples. Whether it was dipped or written with the TNP solution on the paper, the PL of the part stained with TNP was immediately quenched (under irradiation with UV lamp). High background fluorescence arising primarily from paper whitening additives was observed. Therefore, a test paper without further excitation after direct exposure under a UV lamp for 10 min was also used to avoid interferents. In addition, the pattern became increasingly darker as the concentration of TNP in the ink increased. This quenching process is quick, and could be finished within half a minute. These easy-to-use visual techniques could provide a powerful tool for a convenient POC assay for organic pollutants.

\section{Experimental}

\section{Materials and apparatus}

$\mathrm{Al}_{2} \mathrm{O}_{3}$, europium nitrate $\mathrm{Eu}\left(\mathrm{NO}_{3}\right)_{3} \cdot 6 \mathrm{H}_{2} \mathrm{O}, \mathrm{Dy}_{2} \mathrm{O}_{3}$, antibiotic nitrofurazone and $\mathrm{Sr}_{2} \mathrm{O}_{3}$ were purchased from Aladdin Co., Ltd. (Shanghai, China). TNP was obtained from West Long Chemical Co., Ltd. (Sichuan, China). Urea and phosphate buffer solution (PBS, pH 7.4) were obtained from Sigma Co., Ltd. (Missouri, USA). All other reagents were of analytical reagent grade and used without further purification. Doubly distilled water (resistance $>18 \mathrm{M} \Omega \mathrm{cm}^{-1}$ ) was employed throughout all the experiments.

All measurements were carried out at room temperature unless otherwise stated. UV-vis spectroscopic measurements were carried out using a U-3310 spectrophotometer (Hitachi Company, Tokyo, Japan). Luminescence measurements were performed on a microplate reader (Gene Company Limited, USA). PL measurements were carried out on an F-4600 spectrofluorometer (Hitachi Company, Tokyo, Japan). 


\section{Preparation of PLNPs}

Briefly, $\mathrm{Sr}_{2} \mathrm{O}_{3}$ and $\mathrm{Dy}_{2} \mathrm{O}_{3}$ powder were dissolved in concentrated nitric acid to obtain $\operatorname{Sr}\left(\mathrm{NO}_{3}\right)_{2}$ and $\mathrm{Dy}\left(\mathrm{NO}_{3}\right)_{3}$ solution $(0.1 \mathrm{M})$. All other raw materials were dissolved in ultrapure water to obtain a solution with a final concentration of $0.1 \mathrm{M}$. Then, $5 \mathrm{~mL}$ urea, $4 \mathrm{~mL} \mathrm{Sr}\left(\mathrm{NO}_{3}\right)_{2}, 8 \mathrm{~mL} \mathrm{Al}\left(\mathrm{NO}_{3}\right)_{3}, 0.08 \mathrm{~mL} \mathrm{Eu}\left(\mathrm{NO}_{3}\right)_{3} \cdot 6 \mathrm{H}_{2} \mathrm{O}$, and $0.16 \mathrm{~mL} \mathrm{Dy}\left(\mathrm{NO}_{3}\right)_{3}$ were added together to form a solution. The mixture was allowed to react for $1 \mathrm{~h}$. Then, the final solution was adjusted to $\mathrm{pH} 8$ with ammonia. After $2 \mathrm{~h}$, the mixture was transferred into Teflon-lined stainless steel autoclave and heated at $140{ }^{\circ} \mathrm{C}$ for $12 \mathrm{~h}$. After cooling down to room temperature, the obtained product was collected and washed several times with distilled water and absolute ethanol and then dried. The resultant product was directly fired in a zircon crucible in a weak reductive atmosphere $\left[10 \% \mathrm{H}_{2}, 90 \% \mathrm{Ar}\right]$ at $1200{ }^{\circ} \mathrm{C}$ for $10 \mathrm{~h}$. Finally, the powder was ground with an agate mortar and pestle to obtain the desired nanoparticles.

\section{Cell culture}

HeLa cells (cervical cancer cells) were cultured in RPMI 1640 medium supplemented with $10 \%$ fetal bovine serum (FBS) (heat inactivated, GIBCO) and $100 \mathrm{IU} \mathrm{mL} \mathrm{mL}^{-1}$ penicillin-streptomycin (Cellgro) at $37{ }^{\circ} \mathrm{C}$ in a humid atmosphere with $5 \% \mathrm{CO}_{2}$. The cell density was determined using a hemocytometer prior to each experiment.

Initially, $0.5 \mathrm{mg} \mathrm{mL}^{-1}$ PLNPs were incubated with HeLa cancer cells at $37^{\circ} \mathrm{C}$ for $2 \mathrm{~h}$. Then, the old medium was removed and replaced with fresh medium. Confocal microscopy was then used to monitor the results.

MTS experiments were prepared according to the literature. ${ }^{31}$ Cells $\left(5 \times 10^{3}\right.$ HeLa cells per well $)$ were treated with PLNPs in
FBS-free medium. The supernatant medium was replaced after incubation for $2 \mathrm{~h}$ in a cell culture incubator. Then, fresh medium (10\% FBS, $200 \mu \mathrm{L}$ ) was added for further cell growth (48 h). Subsequently, the medium was again removed, and CellTiter reagent $(20 \mu \mathrm{L})$ diluted in fresh FBS-free medium $(100 \mu \mathrm{L})$ was added to each well and incubated for 1-2 h. The absorbance (490 nm) was recorded using a microplate reader. Cell viability was determined according to the manufacturer's description.

\section{Measurement procedure}

In a typical test, $10 \mu \mathrm{L}$ PLNPs solution $\left(1 \mathrm{mg} \mathrm{mL}^{-1}\right)$ and $10 \mu \mathrm{L}$ of different concentrations of target analytes were taken in a centrifuge tube and reacted for $10 \mathrm{~min}$. Then, $180 \mu \mathrm{L}$ PBS buffer was added to the above solution and the total volume of the reaction solution was fixed at $200 \mu \mathrm{L}$.

\section{Results and discussion}

The $\mathrm{Sr}_{2} \mathrm{Al}_{14} \mathrm{O}_{25}: \mathrm{Eu}^{2+}, \mathrm{Dy}^{3+}$ PLNPs were prepared by the hydrothermal-coprecipitation method. Fig. 2 shows the digital photos of the PLNP powder. The deep-green persistent luminescence could be discerned by the naked eyes, revealing a high quantum yield (Fig. 2a). For the PLNPs colloidal solution, transmission electron microscopy (TEM) shows that the asprepared NPs are roughly spherical (Fig. 2b). Dynamic light scattering (DLS) analysis shows a hydrodynamic diameter of $170 \mathrm{~nm}$ (Fig. S1, ESI $\dagger$ ). In order to reveal the spectroscopic properties of the PLNPs colloidal solution, the PL emission and excitation spectra of the PLNPs were investigated. Under excitation wavelengths from $300 \mathrm{~nm}$ to $370 \mathrm{~nm}$, one main fluorescence emission peak at $468 \mathrm{~nm}$ was detected, indicating that
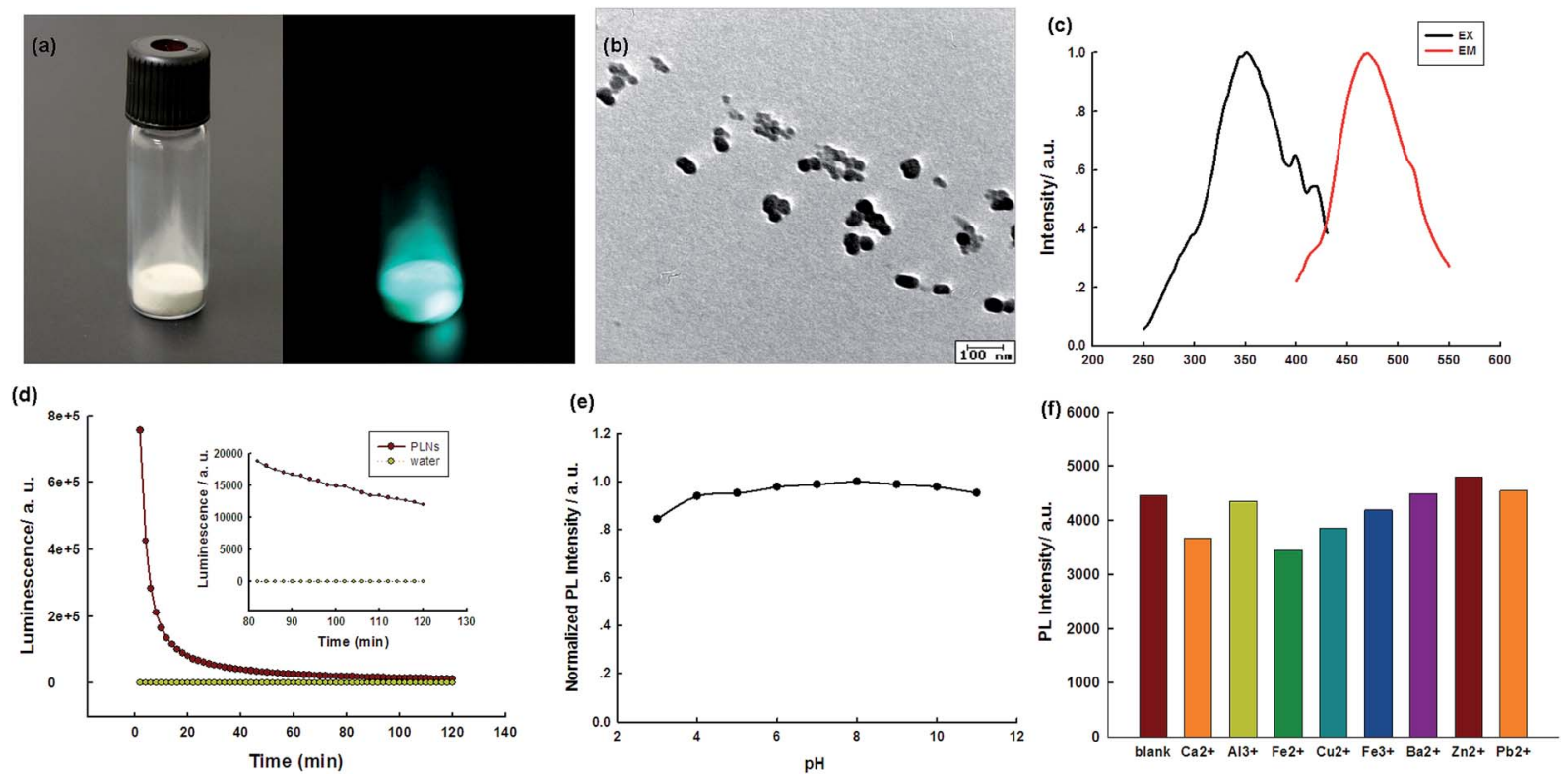

Fig. 2 (a) The digital photos of PLNPs powder (left), and $5 \mathrm{~s}$ after stopping UV irradiation (right); (b) TEM image of PLNPs; (c) excitation and emission spectra of the PLNPs; (d) time-dependent PL intensity of PLNPs (1 mg mL $\left.{ }^{-1}\right)$ without further excitation after direct exposure under a UV lamp for $10 \mathrm{~min}$; (e) PL responses of the PLNPs in different $\mathrm{pH}$ solutions; (f) PL responses of the PLNPs in different media. The concentrations of ions: $10 \mu \mathrm{M}$. 
energy transfer was still effective in water (Fig. S2, ESI $\dagger$ ). A maximum intense peak at $468 \mathrm{~nm}$ was obtained when the excitation wavelength was set at $370 \mathrm{~nm}$ (Fig. 2c and S2, ESI $\dagger$ ). The as-synthesized PLNPs exhibited excellent long-lasting luminescence without further excitation. The PL of asprepared PLNPs lasted $6 \mathrm{~h}$ and became stable in a period of 20 min after excitation (Fig. 2d).

In order to achieve the best sensing performance, the stability of the PLNPs was then studied at different $\mathrm{pH}$ and in the presence of different ions to investigate whether they can work in different conditions. As shown in Fig. 2e, the PL intensity was almost constant from $\mathrm{pH} 3$ to $\mathrm{pH} 10$, indicating that the PLNPs were basically stable at different $\mathrm{pH}$ conditions. The stability of the PLNPs in the presence of several ions was also investigated (Fig. 2f). The PL intensity of the PLNPs was almost negligible in different ionic media, suggesting the possibility of their application in high ionic strength environments.

The sensing platform was first used for antibiotic detection. In the presence of the antibiotic, the PL of PLNPs could be quenched (Fig. S3, ESI $\dagger$ ). The antibiotic nitrofurazone exhibited a wide absorption spectrum from $300 \mathrm{~nm}$ to $480 \mathrm{~nm}$, with a strong absorption peak at $372 \mathrm{~nm}$ (Fig. S4, ESI†). After the addition of TNP into the PLNPs, both their excitation and emission spectra were quenched (Fig. S5, ESI $\dagger$ ). This PL quenching may arise from the potential ET process from the excited PLNPs to the pollutant. ${ }^{\mathbf{1 8 , 3 2}}$ Then, a series of different target concentrations were added to test whether the proposed biosensor could be employed for quantitative detection of the antibiotic. We can observe that the PL intensity gradually decreased with an increase in target concentration. The quenching efficiency could be quantitatively explained by the Stern-Volmer (SV) equation: ${ }^{33,34}$

$$
\left(I_{0} / I\right)=1+K_{\mathrm{sv}}[\mathrm{Q}]
$$

where [Q] is the molar concentration of the analyte, $K_{\mathrm{sv}}$ is the Stern-Volmer constant; $I$ and $I_{0}$ are the PL intensities after and before addition of the analyte, respectively. The change in PL intensity with concentration was linear in the range from 0.025 to $2.5 \mu \mathrm{M}$ (Fig. 3). A limit of detection (LOD) of $5 \mathrm{nM}$ was obtained by $3 \delta /$ slope. Our proposed strategy exhibits shorter assay time and a comparable or even lower, detection limit. ${ }^{\mathbf{1 , 6 , 3 5}}$

To evaluate the selectivity of the PLNPs-based detection system, the responses induced by several potential interferents were also investigated under identical conditions. The obtained results demonstrate that the antibiotic has a high quenching efficiency towards NPs. Other interferents did not cause significant changes in the PL intensity (Fig. S6, ESI $\dagger$ ). In other words, our designed biosensor has a high selectivity towards other molecules.

The PLNPs could also be employed for imaging in living cells. As shown in Fig. $\mathrm{S} 7, \dagger$ the cells incubated with PLNPs show a strong luminescence signal. In addition, the bright-field image confirmed that the cells were viable throughout the imaging experiments. Confocal imaging indicated that the nanoprobe could be imaged in living cells. One key issue for
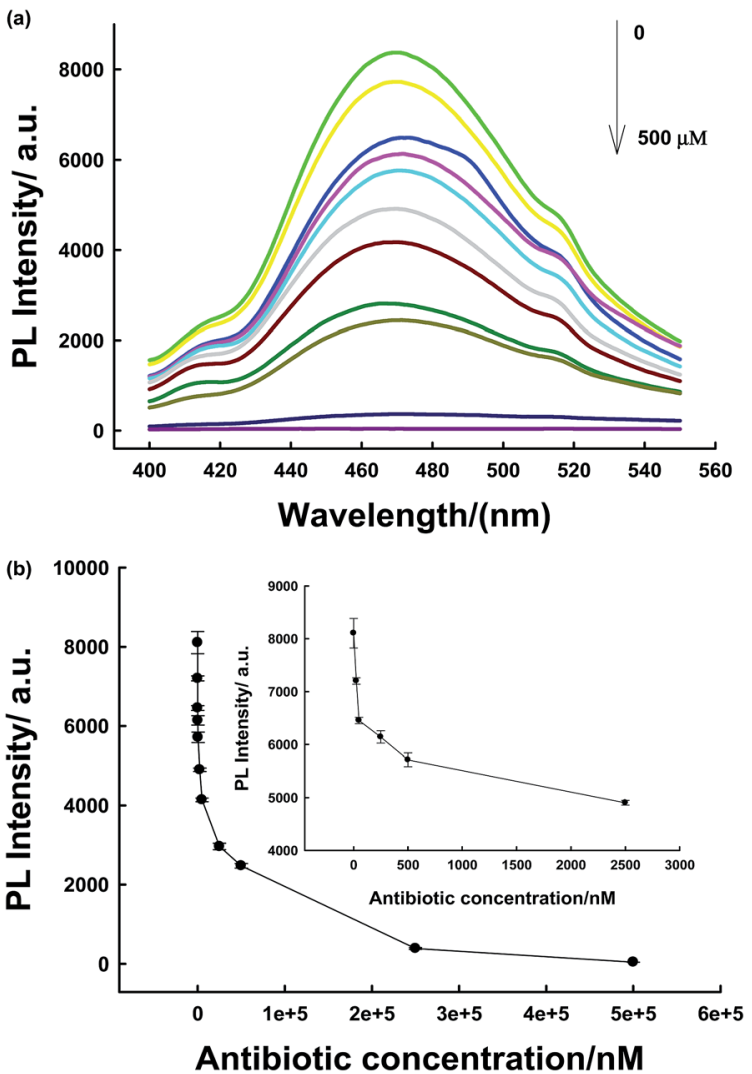

Fig. 3 (a) PL spectra of the assays at various concentrations of antibiotic corresponding to the data in the graph. (b) The relationship between luminescence enhancement and the target concentration. Inset shows the responses of the sensing system to antibiotic at low concentration.

practical bioapplications is the toxicity of PLNPs when employed in bioimaging. ${ }^{33}$ The MTS assay shows that PLNPs have a negligible influence on cancer cell proliferation (Fig. S8†), indicating great prospects for biomedical applications.

The biosensor was then applied in the detection of the antibiotic in real samples (milk). The nanoprobe was first irradiated for $10 \mathrm{~min}$ before detection because the milk solution might produce a strong fluorescent background under in situ excitation. ${ }^{24}$ Therefore, it could eliminate further excitation during the detection process in milk samples and avoid the fluorescent background noise originating from in situ excitation. As shown in Fig. 4a, due to the long-lasting afterglow nature of PLNPs, our proposed sensor could effectively avoid autofluorescence and scattering of light from milk samples under in situ excitation. As shown in Fig. S9, $\uparrow$ the PLNP image showed a dark view after the addition of the antibiotic, while the milk solution with the PLNPs exhibited a bright long-lasting PL in the absence of the target. From the above results, we can conclude that the sensor could be successfully employed to detect antibiotics in complex real samples. Then, the detection range and LOD of the antibiotic in milk were also determined. The detection range was $0.1-50 \mu \mathrm{M}$ in the milk sample (Fig. 4b) with a detection limit of $50 \mathrm{nM}$ at a signal-to-noise ratio of 3 . 

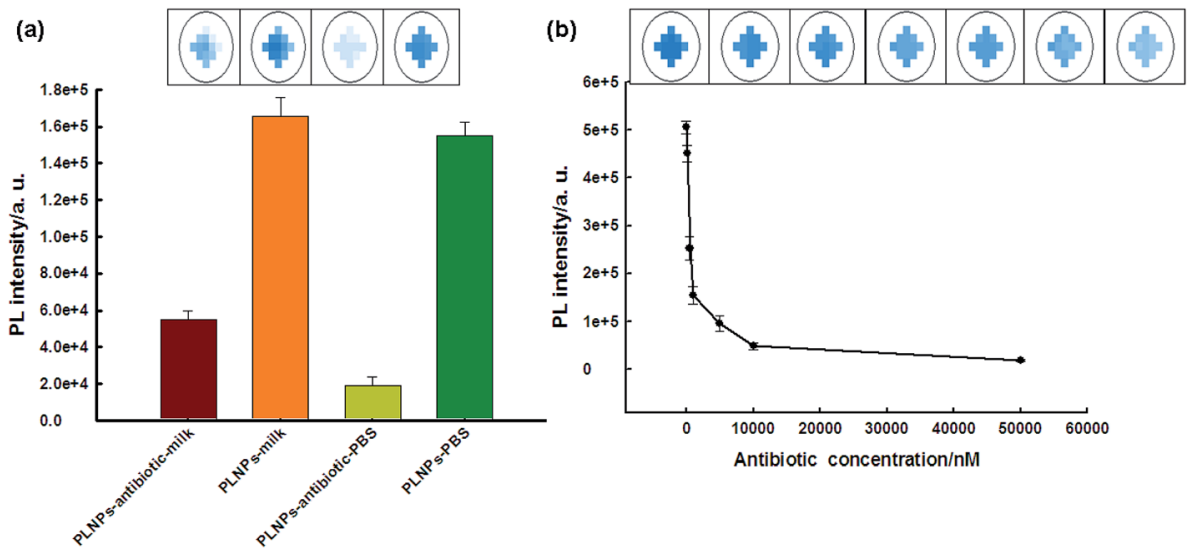

Fig. 4 (a) PL response of milk solutions with antibiotic after in situ excitation ( $365 \mathrm{~nm}$ ) with a UV lamp for 10 min and subsequent removal of the excitation source. The antibiotic concentration was $1 \mu \mathrm{M}$. (b) The relationship between fluorescence enhancement and the target concentration in the real milk samples without further excitation after direct exposure under a UV lamp for $10 \mathrm{~min}$.

TNP has a strong absorption at $370 \mathrm{~nm}$; therefore, there could be a competition for adsorption of excitation light between TNP and PLNPs. Hence, PLNPs could also be employed for the detection of organic explosives based on their PL quenching processes (Fig. S10 and S11†). ${ }^{11}$ As displayed in Fig. 5a, the PL intensity decreased significantly with an increase in the target concentration. The change in intensity exhibited a good relationship within the concentration range of $50 \mathrm{nM}-5$
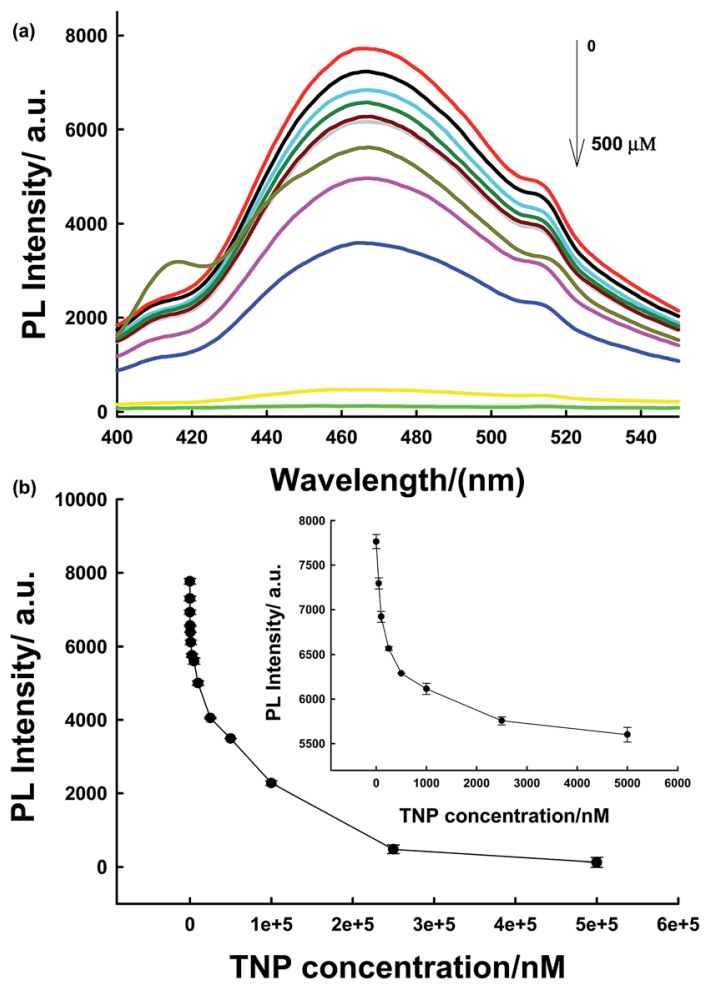

Fig. 5 (a) PL spectra of assay systems at various concentrations of TNP corresponding to data in the graph. (b) The relationship between luminescence enhancement and the target concentration. Inset shows the responses of the sensing system to TNP at low concentration. $\mu \mathrm{M}$ (Fig. 5b). The new system is very sensitive, with a detection limit of $10 \mathrm{nM}$ determined by the $3 \delta /$ slope. Thus, compared to other nitroaromatic detection methods, the PLNPs nanoprobe exhibited a much higher fluorescence quenching response toward TNP (Table S1†). ${ }^{9-11,20,36-39}$ The responses induced by several potential interferents were also studied under the same conditions as those used for TNP detection. The results demonstrate that TNP has high quenching efficiencies toward NFs, but interferents did not induce a visible PL response (Fig. 6).

To further demonstrate the applicability of the TNP sensor, the nanoprobe for the detection of TNP in water samples was investigated. The water samples were obtained from Dianchi Lake (Yunnan, Kunming, China). The water samples were filtered and centrifuged. The recovery experiment was performed on the samples spiked with TNP at different concentration levels because there was no TNP in the water samples detected by the PL sensor. ${ }^{39}$ As shown in Table S2, $\dagger$ it was observed that the real water samples showed good recovery values, suggesting no interferences in these samples. The

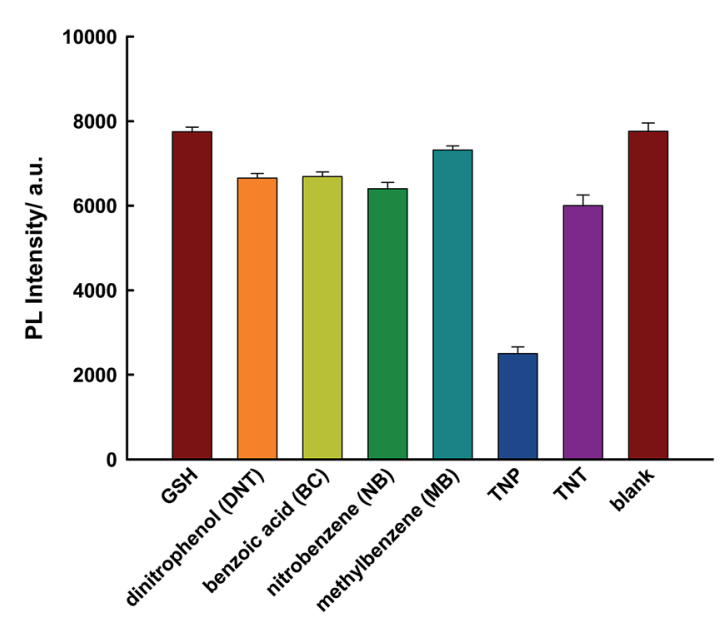

Fig. 6 Specificity for the determination of TNP using the proposed sensor. The concentration of TNP and other interferents is $75 \mu \mathrm{M}$. 
relative standard deviation (RSD) of each sample was below 5\%, indicating that this assay has high reproducibility. Consequently, the proposed PLNPs-based sensor could be successfully applied for the detection of TNP in real water samples.

Then, the nanoprobe sensor was employed for the detection of TNP in the test paper. For test paper assays, a piece of chromatography paper was first inserted into photoluminescent PLNPs colloidal solution. Then, the paper was removed from the solution and dried at $70{ }^{\circ} \mathrm{C}$. The images were recorded by a cell phone. As shown in Fig. 7, a normal paper without any treatment appeared white. Under a $365 \mathrm{~nm}$ UV lamp, the paper exhibited bright blue-green photoluminescence (Fig. 7a). Moreover, the PL intensity increased as the concentration of PLNPs increased (Fig. 7a and b). However, when the word "TNP" was written on the photoluminescent paper using TNP solution, the luminescence of the part stained with TNP was quenched immediately (Fig. 7c and d). In addition, four different concentrations of TNP solutions were dripped on different zones in the paper. Under $365 \mathrm{~nm}$ UV light irradiation, the PL intensity of the four zones was different, and the intensity became increasingly darker when the concentration of TNP was increased. From these abovementioned observations, we can infer that the interference from the background fluorescence arises primarily from paper whitening additives. Therefore, in the following experiment, the treated paper was used without further excitation after direct exposure under a UV lamp for 10 min. As shown in Fig. 7e, a clear and bright luminescence image without any interference from background fluorescence
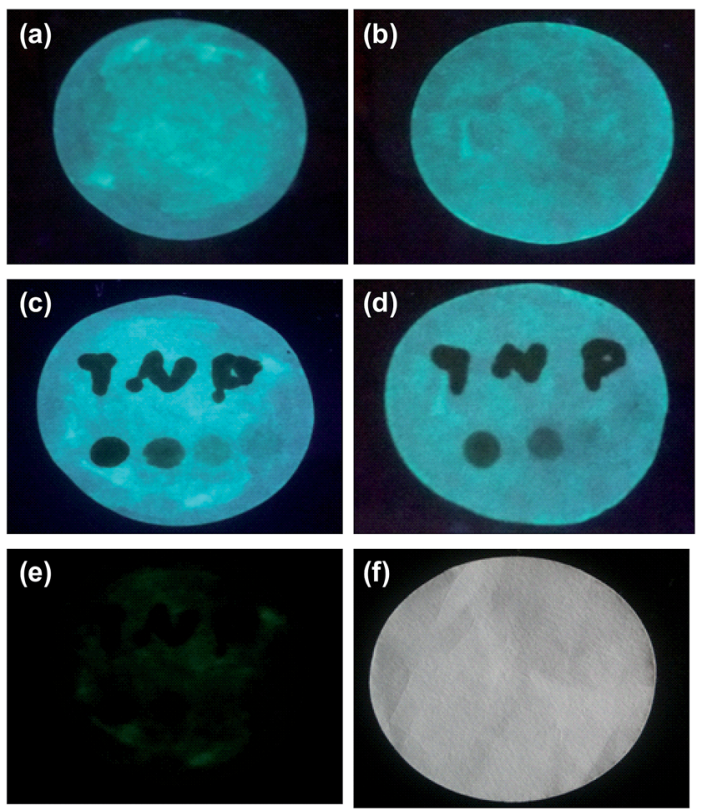

Fig. 7 Photo images of the test paper with high (a) or low concentration of (b) PLNPs treatment; (c and d) visual detection of TNP by handwriting on the treated paper with TNP solution. The concentration of TNP is $0.5 \mu \mathrm{M}, 5 \mu \mathrm{M}, 35 \mu \mathrm{M}$, and $1 \mathrm{mM}$ from pattern under a $365 \mathrm{~nm}$ UV lamp; (e) visual detection of TNP on the treated paper with TNP solution without further excitation after direct exposure under a UV lamp for $10 \mathrm{~min}$; (f) photo image of the paper without any treatment. was observed. The part stained with TNP was still dark, which revealed that the PLNP nanoprobe has the ability to suppress background fluorescence. These properties of PLNPs demonstrate a great potential for latent-finger print imaging, which do not require special equipment. Moreover, the strategy could be integrated with other recognition elements to broaden its applications in bioassays. The above results indicated that our proposed biosensor for the detection of TNP was successfully fabricated.

\section{Conclusion}

In summary, a highly sensitive and label-free photoluminescence sensing platform based on long persistent luminescence nanoparticles was developed for assays of antibiotics and 2,4,6-trinitrophenol. The biosensor was successfully applied for the detection of pollutants in real samples and test papers. Persistent luminescence nanoparticles with long-lasting afterglow could effectively avoid background interference. Under optimal conditions, the limit of detection for TNP was found to be $10 \mathrm{nM}$. The limit of detection for the antibiotic was found to be $5 \mathrm{nM}$ as determined by the $3 \delta /$ slope. The nanoprobe was successfully applied for the detection of pollutants in real samples, i.e., milk and Dianchi Lake water samples. Due to the long-lasting afterglow nature of PLNPs, the signal-to-noise ratio could be greatly increased in complex real samples. By hand writing or finger printing with TNP solution as ink on filter paper soaked with the luminescence nanocomposites, the PL of the part stained with TNP was immediately quenched. Moreover, a test paper without further excitation after direct exposure under a UV lamp for $10 \mathrm{~min}$ was used to avoid interferents. These easy-to-use visual techniques could provide a powerful tool for a convenient POC assay for organic pollutants.

\section{Conflicts of interest}

There are no conflicts to declare.

\section{Acknowledgements}

This study was supported by the National Natural Science Foundation of China (Grants 21605130, 21165023, 21465026, J1210040, 20975034, 21177036, 21275044), and the National Key Scientific Program of China (2011CB911000, 01100205020503104).

\section{References}

1 B. Wang, X. Lv, D. Feng, L. Xie, J. Zhang, M. Li, Y. Xie, J. Li and H. Zhou, J. Am. Chem. Soc., 2016, 138, 6204-6216.

2 L. Lan, Y. Yao, J. Ping and Y. Ying, Biosens. Bioelectron., 2017, 91, 504-514.

3 K. Kümmerer, Chemosphere, 2009, 75, 417-434.

4 Q. Zhang, G. Ying, C. G. Pan, Y. S. Liu and J. L. Zhao, Environ. Sci. Technol., 2015, 49, 6772-6782.

5 E. Song, M. Yu, Y. Wang, W. Hu, D. Cheng, M. T. Swihartc and Y. Song, Biosens. Bioelectron., 2015, 72, 320-325. 
6 M. Han, G. Wen, W. Dong, Z. Zhou, Y. Wu, J. Zhao, D. Li, L. Ma and X. Bu, J. Mater. Chem. C, 2017, 5, 8469-8474.

7 A. Rose, Z. G. Zhu, C. F. Madigan, T. M. Swager and V. Bulovic, Nature, 2005, 434, 876-879.

$8 \mathrm{H}$. He, S. Chen, D. Zhang, E. Yang and X. Zhao, RSC Adv., 2017, 7, 38871-38876.

9 Y. Ma, H. Li, S. Peng and L. Wang, Anal. Chem., 2012, 84, 8415-8421.

10 Y. Q. Xu, B. H. Li, W. W. Li, J. Zhao, S. G. Sun and Y. Pang, Chem. Commun., 2013, 49, 4764-4766.

11 M. Rong, L. Lin, X. Song, T. Zhao, Y. Zhong, J. Yan, Y. Wang and X. Chen, Anal. Chem., 2015, 87, 1288-1296.

12 S. Xing, Q. Bing, H. Qi, J. Liu, T. Bai, G. Li, Z. Shi, S. Feng and R. Xu, ACS Appl. Mater. Interfaces, 2017, 9, 23828-23835.

13 Y. Cui, Y. Yue, G. Qian and B. Chen, Chem. Rev., 2012, 112, 1126-1162.

14 S. S. Nagarkar, B. Joarder, A. K. Chaudhari, S. Mukherjee and S. K. Ghosh, Angew. Chem., Int. Ed., 2013, 52, 2881-2885.

15 Z. Hu, B. J. Deibert and J. Li, Chem. Soc. Rev., 2014, 43, 58155840.

16 C. Zhang, S. Zhang, Y. Yan, F. Xia, A. Huang and Y. Xian, ACS Appl. Mater. Interfaces, 2017, 9, 13415-13421.

17 X. Deng and D. Wu, RSC Adv., 2014, 4, 42066-42070.

18 B. Chen, Z. Liu, H. Zou and C. Huang, Analyst, 2016, 141, 2676-2681.

19 Y. Han, Y. Chen, J. Feng, J. Liu, S. Ma and X. Chen, Anal. Chem., 2017, 89, 3001-3008.

20 P. Huang, W. Zheng, S. Zhou, D. Tu, Z. Chen, H. Zhu, R. Li, E. Ma, M. Huang and X. Chen, Angew. Chem., Int. Ed., 2014, 53, 1252-1257.

21 H. H. Gorris and O. S. Wolfbeis, Angew. Chem., Int. Ed., 2013, 52, 3584-3600.

22 D. Tu, Y. Liu, H. Zhu, R. Li, L. Liu and X. Chen, Angew. Chem., Int. Ed., 2013, 52, 1128-1133.

23 T. Maldiney, A. Bessière, J. Seguin, E. Teston, S. K. Sharma, B. Viana, A. J. J. Bos, P. Dorenbos, M. Bessodes,
D. Gourier, D. Scherman and C. Richard, Nat. Mater., 2014, 13, 418-426.

24 B. Wu, H. Wang, J. Chen and X. Yan, J. Am. Chem. Soc., 2011, 133, 686-688.

25 A. Abdukayum, J. Chen, Q. Zhao and X. Yan, J. Am. Chem. Soc., 2013, 135, 14125-14133.

26 Q. Chermont, C. Chanéac, J. Seguin, F. Pellé, S. Maîtrejean, J. Jolivet, D. Gourier, M. Bessodes and D. Scherman, Proc. Natl. Acad. Sci. U. S. A., 2007, 104, 9266-9271.

27 N. Li, Y. Li, Y. Han, W. Pan, T. Zhang and B. Tang, Anal. Chem., 2014, 86, 3924-3930.

28 Z. Pan, Y. Lu and F. Liu, Nat. Mater., 2012, 11, 58-63.

29 D. Tu, L. Liu, Q. Ju, Y. Liu, H. Zhu, R. Li and X. Chen, Angew. Chem., Int. Ed., 2011, 50, 6306-6310.

30 Y. Liu, D. Tu, H. Zhu and X. Chen, Chem. Soc. Rev., 2013, 42, 6924-6958.

31 R. Hu, X. Zhang, Z. Zhao, G. Zhu, T. Chen, T. Fu and W. Tan, Angew. Chem., Int. Ed., 2014, 126, 5931-5936.

32 X. Hu, T. Wei, J. Wang, Z. Liu, X. Li, B. Zhang, Z. Li, L. Li and Q. Yuan, Anal. Chem., 2014, 86, 10484-10491.

33 R. M. Jones, T. S. Bergstedt, D. W. McBranch and D. G. Whitten, J. Am. Chem. Soc., 2001, 123, 6726-6727.

34 L. Chen, D. W. McBranch, H. Wang, R. Helgeson, F. Wudl and D. G. Whitten, Proc. Natl. Acad. Sci. U. S. A., 1999, 96, 12287-12292.

35 H. Chen, B. Wang, Ji. Zhang, C. Nie, F. Lv, L. Liu and S. Wang, Chem. Commun., 2015, 51, 4036-4039.

36 Y. Liu, S. Zhou, D. Tu, Z. Chen, M. Huang, H. Zhu, E. Ma and X. Chen, J. Am. Chem. Soc., 2012, 134, 15083-15090.

37 G. Das, B. P. Biswal, S. Kandambeth, V. Venkatesh, G. Kaur, M. Addicoat, T. Heine, S. Verma and R. Banerjee, Chem. Sci., 2015, 6, 3931-3939.

38 D. Chen, N. Zhang, C. Liu and M. Du, ACS Appl. Mater. Interfaces, 2017, 9, 24671-24677.

39 Y. Wang and Y. Ni, Anal. Chem., 2014, 86, 7463-7470. 